\section{Changement climatique et agricultures du monde}

Emmanuel Torquebiau, éditeur scientifique. 2015, Changement climatique et agricultures du monde. Ed. Quae, 328 pp., http://www.quae.com/fr/r4103-changement-clima tique-et-agricultures-du-monde.html.

L'objectif de cet ouvrage est de faire le point sur les liens entre changement climatique et agriculture des pays du Sud (y compris élevage et foresterie) en mêlant études de cas et travaux de synthèse.

Les analyses présentées se situent dans le contexte du changement climatique tel qu'exposé dans les documents du $5^{\mathrm{e}}$ Rapport d'évaluation du Giec de septembre 2013 à octobre 2014.

Une introduction expose les grandes tendances du changement climatique et la façon dont il modifie la donne agricole.

La première partie traite, sur la base d'exemples, de la réponse aux stress climatiques qui sont la chaleur, la salinité, la sécheresse, les bio-agresseurs et les maladies. Une analyse au niveau des systèmes de culture et d'élevage met en évidence la nécessité de prendre en compte dans un certain nombre de cas des combinatoires de différents stress.
La deuxième partie est consacrée à des exemples d'agriculture et d'élevage " climato-intelligents » ainsi qu'à des résultats expérimentaux sur de nouvelles pratiques et à des innovations des agriculteurs. Ils mêlent adaptation et atténuation parfois au sein d'une même pratique telle que par exemple l'agroforesterie.

La troisième partie fournit des outils d'évaluation (analyse de cycle de vie) et propose des mesures susceptibles de stimuler les transformations vers des pratiques climato-intelligentes : services écosystémiques, certifications et labels, politiques publiques, etc.

La quatrième partie projette l'agriculture climato-intelligente dans l'espace en la resituant dans les arènes de la négociation internationale et dans le temps en déclinant de nouvelles perspectives de recherche au regard des enjeux climatiques concernant les agricultures du Sud.

Les auteurs de l'ouvrage ont délibérément privilégié globalement des exemples d'options techniques et des instruments politiques et économiques permettant de faire des arbitrages relativement à la problématique du changement climatique.

La cible de l'ouvrage concerne les chercheurs, les ingénieurs, les opérateurs divers du secteur agricole, forestier et de l'élevage dans les pays du Sud, les étudiants et le public averti.

Jérôme Lazard (jerome.lazard@cirad.fr) 